\title{
Significance of positive surgical margin and how to minimize in robotic radical prostatectomy
}

\begin{abstract}
Radical removal of the tumor is the key for a successful curative cancer surgery. The target of radical prostatectomy [RP] was to completely resect the tumor. Positive surgical margins [PSM] following RP are associated with high risks of biochemical recurrence $[\mathrm{BCR}]$ and secondary treatment. This literature highlights the significance of PSM, how to manage and how the robotic radical prostatectomy could prevent it. It also evaluates the currently available literature to spot the PSM-associated factors and how to manage them, the characters of PSM including number, size, site, Gleason score at the margin and their role in the recurrence, lastly, the novel imaging and surgical approaches that may reduce or eliminate the risk of PSM in the future.
\end{abstract}

Keywords: Biochemical recurrence; Radical prostatectomy; Positive surgical margins; Robotic radical prostatectomy
Volume 6 Issue 4 - 2018

\author{
Abdalla Deb,' Rajiv Pillai \\ 'Senior Clinical Fellow in Urology, Colchester University \\ Hospital, UK \\ ${ }^{2}$ Consultant Urologist, Colchester General University Hospital, \\ UK
}

Correspondence: Dr. Abdalla Ali Abdel Hamed Ali Deb, Senior Clinical Fellow in Urology, Colchester University Hospital, Colchester, UK, Tel +4407456070200,

Email drabdodeeb@hotmail.com

Received: June 15, 2018| Published: August 28, 2018
Abbreviations: ADT, androgen deprivation therapy; AJCC, american joint commission on cancer; ASTRO, american society for therapeutic radiology organization; AUA, american urological association; BCR, biochemical recurrence; EPE, extra-prostatic extension; NVB, neurovascular bundle; PCa, prostate cancer; PSM, positive surgical margin; QoL, quality of life; RALRP, robot-assisted laparoscopic radical prostatectomy; RARP, robot-assisted radical prostatectomy; RP, radical prostatectomy; SM, surgical margins

\section{Introduction}

Prostate cancer is the 2nd leading cause of cancer-related deaths in males. Its incidence is rising as a result of the improved life expectancy and the increased PSA screening. ${ }^{1}$ There are several treatment approaches for prostatic cancer, one of the main approaches for the localized type $(\mathrm{PCa})$ is the radical prostatectomy [RP] which offers a high 5-year disease-specific survival rate of $>95 \% .{ }^{1}$ The contemporary RP series hold variable, technique-independent, positive surgical margin [PSM] rates [10-48\%] while robot-assisted RP holds higher posterolateral PSM rates $[52.3 \%] .^{2}$

These variable rates have been attributed to many factors including the complex prostatic anatomy, the surgeon's experience, and the use of non-standardized pathological techniques \& definitions. Unfortunately, an exact cause has not been identified yet. A recent study reported that the type of surgery used only modulates the site of the PSMs and not the rate of occurrence; it also claimed that a high rate of apical PSM [38.5\%] was encountered with the open RP. ${ }^{2}$

The presence of PSMs following RP reflects the incomplete cancer excision and has been associated with a shorter time to progression, a high rate of BCR, and requires a complementary therapy such as androgen deprivation therapy or radiotherapy. ${ }^{3}$

The main surgical treatment for prostatic cancer in the United States of America is the Robot-assisted radical prostatectomy [RARP] which accounts for over $80 \%$ of all RPs done in $2013 .{ }^{4}$ RARP facilitates nerve-sparing procedures with better postoperative continence and potency. ${ }^{5}$ However, sparing the neurovascular bundles decrease the safety distance between the cancerous tissue and the surgical margins which is associated with an increased incidence of PSMs. ${ }^{6}$

\section{Definition of surgical margin}

In theory, a PSM is defined as clear; "tumor that extends to the surface of the prostate wherein the surgeon has cut across the tissue plane". ${ }^{7}$ However, since there is no true histologic capsule around the prostate, defining surgical margins in practice could be challenging. In order to facilitate defining surgical margins [SM] status upon receipt by the pathologist, the entire surgical specimen should be inked and fixed. A positive margin is simply identified as "cancer cells extending to the inked surface of the specimen". Margin status is negative if tumor cells are microscopically close to $[<0.1 \mathrm{~mm}]$, but not actually in contact with the inked surface or when present at the surface of the non-inked tissue. ${ }^{8}$

Even with proper handling of the specimen by the pathologist, SM assessment may be complicated by incomplete or irregular tracking of ink, crush, thermal, or electrocautery artefact and partial tearing of the extraprostatic soft tissue during processing or tissue banking. ${ }^{9}$

Surgical margins in the presence of extra-prostatic extension [EPE] may represent an over enthusiastic surgeons' effort to spare the neurovascular bundle [NVB] or tumor that invades into vital structures and could not be completely resected. PSM in the absence of EPE, usually represent a capsular incision into tumor, an iatrogenic positive margin, due to an improper dissection plane with incision into the prostate and into the tumor. ${ }^{10}$

\section{Characteristic of surgical margin}

A high risk of BCR is encountered in cases having PSM. ${ }^{11}$ Many researchers have been trying to clarify the pathological characters of PSM in order to better risk stratify patients and potentially offer an 
adjuvant intervention for those at a high-risk of progression while sparing unnecessary adjuvant treatment for others. In summary, these data suggest that the length of the PSM, the number of PSMs, the Gleason score at the PSM, and potentially even the location of the PSM may each play an important role in defining the risk of BCR following RP.

\section{Amount" of positive margin}

Multiple investigators have sought to quantify the "amount" of PSM either by counting the number of positive margins in a given specimen, or the extent of the positive margin quantified as binary variable such as focal versus extensive often seen in older studies or as a more reproducible linear extent. The rationale behind these attempts assumes that a greater amount of PSM is associated with a greater quantity of tumor left behind and a greater potential for growth, biochemical recurrence, and metastases. Although the number of positive margins may be an independent predictor in multivariable analysis for BCR, the number of positive margins may not significantly impact the predictive accuracy of nomogram predictions compared to a PSM modeled more simply as positive or negative. ${ }^{11}$

\section{Anatomic location of positive surgical margin}

Repeatedly studies have demonstrated that the two most likely locations for PSM are the apex of the prostate and the posterolateral margins. Together these sites make up the majority of PSM accounting for $60-75 \%$ of PSM in most reported series of either open retropubic or robotic approaches. ${ }^{12}$ The apex of the prostate contains less supporting tissue than the rest of the gland with the least amount of capsule, and even benign glands can become admixed with skeletal muscle at this location. This coupled with the increased traction placed on the apex during various parts of the procedure and efforts to maintain urethral length may account for the heightened incidence of PSM at this location. The prostatic posterolateral margin is the 2 nd most common location of PSMs and this is likely due to attempts to preserve as much of the neurovascular bundle as possible which run in this location. ${ }^{13}$

\section{Gleason score at positive surgical margin}

Theoretically when a higher Gleason score is found at the PSM, a more aggressive tumor remains in the patient with potentially higher rates of BCR. Several studies have demonstrated that grade of cancer at the PSM is associated with greater rates of BCR. ${ }^{14}$ Gleason score in the primary tumor is highly correlated with Gleason score at the margin for Gleason 6 tumors but this concordance rate diminishes rapidly as the primary tumor Gleason score increases. ${ }^{14}$

\section{Types of positive surgical margin}

Robotic urologic surgery, a newly emerging technique with tremendous potentials. Robotic-assisted laparoscopic radical prostatectomy [RARP] has becoming a popular surgical treatment for the clinically localized prostatic cancers. ${ }^{15}$

Two variants of positive surgical margins have been identified including iatrogenic and non-iatrogenic. ${ }^{16}$ The iatrogenic positive margins, which could be simply avoided by widening the dissection margin, are caused by capsular incision in organ-confined tumors $[\mathrm{pT} 2+]$, or cutting across an area of extraprostatic tumor extension. ${ }^{17}$

While the non-iatrogenic margins are caused by the extension of the cancerous tissue through the capsule and periprostatic tissue, to the edge of the surgical specimen making a complete tumor resection is inapplicable as it will lead to unacceptable morbidities [eg, cutting through the rectal wall]. Both iatrogenic from non-iatrogenic margins are pathologically distinguishable, except for $\mathrm{pT} 2+$ cases with a capsular gap in which the extraprostatic tumor extension is difficult to ascertain. ${ }^{17}$

\section{Reducing positive surgical margins rates}

It is highly essential for the urologist to do their best to minimize the risk of PSMs, while maintaining the best possible postoperative urinary and erectile function, because they majorly distress the patients and hold a possibility of requiring a complementary treatment. ${ }^{18}$

\section{Surgical experience}

It has been proven that the rate of PSMs is related to the surgeon's experience as the least positive margins are usually encountered with the highly experienced surgeons. Surgical learning curve highlighting the role of the surgeon's experience in the surgical outcome has been developed for open RP, laparoscopic RP, and RALRP. ${ }^{[19-21]}$ They showed an initially high rate of PSMs that gradually levels off with accumulating experience. The number of surgeries required to achieve that lowest rate of PSMs ranges between 200-250 surgeries in the laparoscopic series ${ }^{20}$ to $1000-1500$ robotic surgeries. ${ }^{21}$

Being experienced in open or laparoscopic RP and attending a fellowship training greatly expedite the transition to the robotic interface and reduce the incidence of the associated PSMs. ${ }^{22}$

\section{Bladder neck margin}

Extraprostatic extension with microscopic invasion of the bladder neck-previously designated as T4 according to the American joint commission on cancer [AJCC]-has recently been revised to be included in T3a category. This reclassification is based on the work of several retrospective series which have demonstrated that patients with isolated positive bladder neck margins have outcomes that more closely approximate $\mathrm{T} 3$ lesions. ${ }^{23}$

It is unclear whether a PSM at the bladder neck means a worse prognosis than PSM in other locations, as isolated bladder neck margins are rare and often associated with multiple high-risk features. ${ }^{24}$ Further investigation is needed to clearly define whether isolated bladder neck margin truly does have a worse prognosis than margins in other locations, if confirmed, clarification of the AJCC might be to define bladder neck invasion as $\mathrm{T} 3 \mathrm{~b}$ and seminal vesicle invasion as $\mathrm{T} 3 \mathrm{c}$ as suggested by some groups. ${ }^{23}$

\section{Surgical approach}

With the huge shift towards robotics and away from open RP that has occurred over the last decade, the pertinent question is whether surgical approach has an impact on the SM status. ${ }^{25}$

In a recently published meta-analysis with propensity adjustment for patient, surgeon, and hospital factors, the authors found no difference in PSM for open and robotic surgery. ${ }^{26}$ A prior meta-analysis that limited its analysis to comparative studies only demonstrated that PSM rates were similar between approaches. ${ }^{27}$ Administrative care datasets have not been able to directly compare PSM for differing surgical approaches but have demonstrated similar rates in the use 
of secondary therapies between different surgical approaches as a surrogate. $^{28}$

\section{Technical modification during robot-assisted laparoscopic radical prostatectomy}

One of the reliable methods, adopted by surgeons, to determine the safe resection margin during open surgery is using tactile feedback which is based on the fact instating that tumor-infiltrated tissue is elastically different from the normal tissue, such method has been proven to minimize the incidence of PSMs. Although, robotic surgeries lack this advantage, they overcome this obstacle by the ability to delineate key anatomic landmarks via the intraoperative visual cues. ${ }^{29}$

\section{Apical margins}

Precise dissection of the apex is a major challenge faced during RP as the apex is not easily accessible being deeply located under the pubic arch near to vital structures such as the dorsal venous complex, erectile nerves, rectum, and sphincter. Also, the highly variable configuration resulting from the asymmetrical appearance of some glands and the possible posterior protrusion in the urethra caused by some other glands, known as the posterior apical notch, as well as, lacking a distinct capsule along with the periprostatic tissues located on the posterolateral surface of the prostate make it difficult to detect accurate tumor-free dissection planes. The concealed posterior apical notch might be violated during surgery, especially if the dissection preformed in a plane perpendicular to the axis of the urethra. Several attempts have been made to discover the optimum dissection technique of the prostatic apex in order to preserve both sexual and urinary functions postoperatively without compromising the surgical margin. $^{30}$

\section{Posterolateral margins}

The trials to preserve the postoperative erectile functions via nerve sparing during tumor resection carry a serious possible risk PSMs, irrespective of surgical technique, because of the close contact between the prostate and surrounding neurovascular tissue. The PSMs could result from iatrogenic intraprostatic incision into an otherwise organ confined tumor or failure to excise the extraprostatic extension of the prostate carcinoma. ${ }^{31}$

Usually, the incidence of PSMs is higher with apical resection than the posterolateral prostatic resection in stage pT2 and pT3 disease. Such higher rates were attributed to the absence of the capsule anteriorly, the interlocking of the apex and the striated muscle of the external sphincter, the insufficient mobilization of the fibromuscular bands that tether the apex and the possibility of limited visibility and accessibility caused by the pubic bone, anterior prostatic fat, or bleeding from the dorsal venous complex. ${ }^{32}$

To guarantee a complete tumor dissection, some cases may require dissection in more than one posterolateral plane, thus, nerve preservation should not be considered an all-or-none phenomenon. It is vital to use all the available preoperative assessment tools, such as clinical biopsy data, rectal examination, endorectal magnetic resonance imaging, to determine the exact outlines of the mass in order to deliver the best dissection and avoid PSMs. ${ }^{33}$

According to the results of the thorough preoperative evaluation, surgeons can effectively determine the most appropriate dissection plane which could completely preserve the nerve guided by glistening view of the prostate as in the intrafascial plane, partially preserve the nerve as in the interfascial plane or does not preserve the nerve at all as in extrafascial plane. The criteria of the mass is the main determinant of the dissection plane, a more advanced tumor requires the use of the interfascial and extrafascial planes and is more liable to technical errors as forcing a plane with blunt dissection is prone to produce a capsular flap at areas of adhesions or entry of capsular arteries]. ${ }^{34}$

\section{Frozen-section analysis}

Another reliable technique to ascertain the choice of the best dissection plane is performing an intraoperative frozen-section histological evaluation to ensure safe surgical margins. ${ }^{35}$ However, this technique was considered of no value and still has not proven to be a necessity for all patients. Early research evaluated the value of using frozen-section histological assessment during RP only when the surgeon was suspicious about a specific area and the results were not encouraging. ${ }^{36}$

Over the recent years, a thorough assessment of the whole prostate has been suggested as a systemic approach for a safe resection. Schlomm and colleagues reported that the systemic frozen section analysis substantially reduce the incidence of PSMs and safely spare the nerve in high-risk patients who would otherwise have been considered candidates for non-nerve-sparing approach, thus, according to their conclusion, it is a useful adjunct to the surgical preplanning. ${ }^{37}$

However, more studies are still needed to confirm the long-term oncologic and functional benefits of this approach. It is essential to refer to an important observation documented by the aforementioned study which demonstrated a lower incidence of cancer (about 25\%) detected by the histopathological analysis of secondary-excised specimens when the margin was deemed positive by frozen section study. The high false-negative rate reported by the same study could be attributed to the fact that secondary resection was not performed exactly at the corresponding anatomic location. On the other hand, malignant cells contacting the inked surface [PSMs] do not necessarily indicate that cancer was left behind. Obviously, the latter condition has explicit implications for secondary therapy. ${ }^{34}$

\section{Imaging tools predict or prevent positive margins}

\section{a) Pre-operative MRI}

MRI has been demonstrated to alter surgical plan prior to RP in approximately $40 \%$ of patients; however, it has significant interobserver variability. ${ }^{38}$

\section{b) A real-time transrectal ultrasound}

A real-time transrectal ultrasound during RP to help outline the suspected area of EPE with a reduction in their PSM rates from $29 \%$ to $9 \% .{ }^{39}$

More recently urologists have begun to incorporate the use of a transrectal ultrasound probe with concurrent use of the TilePro to display the ultrasound images on the da Vinci surgical system console. Mounting interest in MR-US fusion technology is likely to result in 
utilizing this technology in a similar fashion to attempt to minimize PSM and maximize preservation of the NVB. ${ }^{40}$

\section{c) Near-infrared fluorescence imaging}

Near-infrared fluorescence imaging has been used for the identification of renal tumors ${ }^{34}$ and sentinel lymph nodes for prostate cancer. $^{35}$

\section{Management of positive margins after radical prostatectomy}

Large multi-institutional studies have demonstrated that patients with PSM are more than twice as likely to experience BCR as patients without, even after adjusting for age, PSA, pathologic Gleason score, pathologic stage, and year of surgery. This leaves clinicians and patients in the challenging position of considering the role for additional treatment in the absence of any detectable disease. Unfortunately, adjuvant radiotherapy comes at the cost of increased risk of urinary incontinence, urinary stricture disease, proctitis, and rectal bleeding. Furthermore, although patients with PSM are at an increased risk of developing BCR many never do and are exposed to the harms of adjuvant radiotherapy without benefit. ${ }^{11}$

Three randomized trials potential have examined the role of adjuvant radiotherapy in men with 'adverse' pathologic features in the RP specimen. ${ }^{3,41,42}$ All documented improvement in BCR free-survival with adjuvant radiotherapy compared to a "wait and see' approach. Based largely on the results of these three trials the American Urological Association [AUA] and the American Society for Therapeutic Radiology Organization [ASTRO] released joint guidelines stating that patients with adverse pathologic features [including but not limited to a PSM] should be offered ART. ${ }^{43}$

The guidelines continue on to state that the decision of whether to receive adjuvant radiotherapy should be based on a shared decision-making process by a multidisciplinary team and the patient, with consideration of "patient's history, functional status, values, preferences, and tolerance for potential toxicities and QoL effects of radiotherapy."

It has been demonstrated that salvage therapy administered at lower PSA levels is associated with greatest effectiveness. The ability to detect PSA at very low levels has led many to conclude that a preferable strategy would be to offer early salvage treatment when patients have low but detectable PSA rather than adjuvant radiotherapy. Such a strategy may reduce the over treatment of patients who are never destined to develop BCR while maintaining the potential advantage of radiotherapy.

Lastly, some practitioners use androgen deprivation therapy [ADT] alone for patients with adverse pathologic characteristics including a PSM. In one small randomized trial, whose results have not been confirmed, ADT following prostatectomy for patients with lymph node positive disease was demonstrated to result in overall survival benefit but for node negative patients ADT has never been demonstrated to have similar benefit. ADT has the potential for significant harm, reduces QoL, and should only be considered for patients with a positive lymph node or those undergoing adjuvant or salvage radiotherapy. ${ }^{44}$

\section{Conclusion}

PSM are associated with an increased risk of BCR. The presence of a PSM may be more influenced by the tumor biology [volume, distribution, and aggressiveness] and by the individual surgeon's experience than the surgical approach [type of procedure and technique] used to perform RP. Longer PSM [ $>3 \mathrm{~mm}]$, multiple PSM, and higher Gleason score at the PSM are associated with an increased likelihood of BCR, while isolated apical PSM have a lower risk of BCR. Despite the fact a PSM at the posterolateral location indicates the highest relapse probability, the prognosis of the apical PSMs is not yet certain. The surgeon's experiences along with attention to details are vital in the reduction of PSMs rate whatever is the adopted surgical technique [open, laparoscopic, or robotic]. Preoperative planning with eMRI and intraoperative frozen section analysis may reduce the rate of PSMs especially in high-risk cases. RT is the only established therapy curative potential in cases with PSM on final pathology.

Robotic prostatectomy has been proven to offer a better shortterm oncological control, urinary and sexual functions in comparison to the open radical prostatectomy. Surgeons who are experienced in RARP have a lower rate of PSMs. RARP can provide comparable surgical margin results for cases with both low- and high-risk disease. Although RARP lacks the benefits of tactile sensation, such issue is overcome by a combination of thorough preoperative assessment, clinical algorithms for excision of the NVB, intraoperative visual clues, and surgeon's experience. Surgical margin rates provide the best surgeon-related surrogate endpoint to predict the prognosis following radical prostatectomy till now.

\section{Acknowledgements}

None.

\section{Conflict of interest}

None.

\section{References}

1. Parkin DM, Bray F, Devesa S. Cancer burden in the year 2000. The global picture. Eur J Cancer. 2001;3(S8):S4-66.

2. Magheli A, Busch J, Leva N, et al. Comparison of surgical technique (open vs. laparoscopic) on pathological and long term functional outcomes following radical prostatectomy. BMC Urol. 2014;14:18.

3. Bolla M, van Poppel H, Collette L, et al. Postoperative radiotherapy after radical prostatectomy: a randomised controlled trial (EORTC trial 22911). Lancet. 2005;366(9485):572-578.

4. Skarecky DW. Robotic-assisted radical prostatectomy after the first decade: surgical evolution or new paradigm. ISRN urology. 2013;157379:1-22

5. Ficarra V, Novara G, Ahlering TE, et al. Systematic review and metaanalysis of studies reporting potency rates after robot-assisted radical prostatectomy. Eur Urol. 2012;62(3):418-430.

6. Coelho RF, Rocco B, Patel MB, et al. Retropubic, laparoscopic, and robot-assisted radical prostatectomy: a critical review of outcomes reported by high-volume centers. J Endourol. 2010;24(12):2003-2015.

7. Tan PH, Cheng L, Srigley JR, et al. International Society of Urological Pathology (ISUP) consensus conference on handling and staging of radical prostatectomy specimens. Working group 5: surgical margins. Mod Pathol. 2011;24(1):48-57.

8. Emerson RE, Koch MO, Daggy JK, et al. Closest distance between tumor and resection margin in radical prostatectomy specimens: lack of prognostic significance. Am J Surg Pathol. 2005;29(2):225-229. 
9. Evans AJ, Henry PC, Van der Kwast TH, et al. Interobserver variability between expert urologic pathologists for extraprostatic extension and surgical margin status in radical prostatectomy specimens. Am J Surg Pathol. 2008;32(10):1503-1512.

10. Meeks JJ, Eastham JA, Radical prostatectomy: positive surgical margins matter. Urologic Oncology: Seminars and Original Investigations. 2013;31(7):974-979

11. Stephenson AJ, Wood DP, Kattan MW, et al. Location, extent and number of positive surgical margins do not improve accuracy of predicting prostate cancer recurrence after radical prostatectomy. $J$ urol. 2009;182(4):1357-1363.

12. Smith JA Jr, Chan RC, Chang SS, et al. A Comparison of the Incidence and Location of Positive Surgical Margins in Robotic Assisted Laparoscopic Radical Prostatectomy and Open Retropubic Radical Prostatectomy. $J$ Urol. 2007; 178: 2385-2390.

13. Voges GE, McNeal JE, Redwine EA, et al. Morphologic analysis of surgical margins with positive findings in prostatectomy for adenocarcinoma of the prostate. Cancer. 1992;69(2):520-526.

14. Udo K, Cronin AM, Carlino LJ, et al. Prognostic impact of subclassification of radical prostatectomy positive margins by linear extent and Gleason grade. J urol. 2013;189(4):1302-1307.

15. Atug F, Castle EP, Srivastav SK, et al. Positive surgical margins in robotic-assisted radical prostatectomy: impact of learning curve on oncologic outcomes. Eur urol. 2006;49(5):866-872.

16. Epstein JI, Amin M, Boccon-Gibod L, Egevad L, Humphrey PA, Mikuz $\mathrm{G}$, et al. Prognostic factors and reporting of prostate carcinoma in radical prostatectomy and pelvic lymphadenectomy specimens. Scand J Urol Nephrol Suppl. 2005;39(216):34-63.

17. Yossepowitch O, Bjartell A, Eastham JA, et al. Positive surgical margins in radical prostatectomy: outlining the problem and its long-term consequences. Eur urol. 2009;55(1):87-99.

18. Hong YM, Hu JC, Paciorek AT, et al. Impact of radical prostatectomy positive surgical margins on fear of cancer recurrence: Results from CaPSURETM . Urologic Oncology: Seminars and Original Investigations. 2010;28(3):268-273.

19. Ponholzer A, Madersbacher S. Re: The learning curve for surgical margins after open radical prostatectomy: implications for the use of margin status as an oncologic endpoint. Eur urol. 2011;59(1):171-172.

20. Secin FP, Savage C, Abbou C, et al. The learning curve for laparoscopic radical prostatectomy: an international multicenter study. $J$ Urol. 2010;184(6):2291-2296.

21. Sooriakumaran $\mathrm{P}$, John M, Wiklund P, et al. Learning curve for robotic assisted laparoscopic prostatectomy: a multi-institutional study of 3794 patients. Minerva Urol Nefrol. 2011;63(3):191-198.

22. Wolanski P, Chabert C, Jones L, et al. Preliminary results of robot $\square$ assisted laparoscopic radical prostatectomy (RALP) after fellowship training and experience in laparoscopic radical prostatectomy (LRP). BJU int. 2012;110(S4):64-70.

23. Pierorazio PM, Epstein JI, Humphreys E, Han M, Walsh PC, Partin AW. The significance of a positive bladder neck margin after radical prostatectomy: the American Joint Committee on Cancer Pathological Stage T4 designation is not warranted. J Urol. 2010;183(1):151-157.

24. Yossepowitch O, Sircar K, Scardino PT, et al. Bladder neck involvement in pathological stage pT4 radical prostatectomy specimens is not an independent prognostic factor. J Urol. 2002;168(5):2011-2015.

25. Lowrance WT, Eastham JA, Savage C, et al. Contemporary open and robotic radical prostatectomy practice patterns among urologists in the United States. J Urol. 2012;187(6):2087-2092.
26. Tewari A, Sooriakumaran P, Bloch DA, et al. Positive surgical margin and perioperative complication rates of primary surgical treatments for prostate cancer: a systematic review and meta-analysis comparing retropubic, laparoscopic, and robotic prostatectomy. Eur Urol. 2012;62(1):1-15.

27. Ficarra V, Novara G, Artibani W, et al. Retropubic, laparoscopic, and robot-assisted radical prostatectomy: a systematic review and cumulative analysis of comparative studies. Eur Urol. 2009;55(5):1037-1063.

28. Hu JC, Gu X, Lipsitz SR, et al. Comparative effectiveness of minimally invasive vs open radical prostatectomy. JAMA. 2009;302(14):1557-1564.

29. Patel VR, Schatloff O, Chauhan S, et al. The role of the prostatic vasculature as a landmark for nerve sparing during robot-assisted radical prostatectomy. Eur Urol. 2012;61(3):571-576.

30. Tewari AK, Srivastava A, Mudaliar K, et al. Anatomical retro $\square$ apical technique of synchronous (posterior and anterior) urethral transection: a novel approach for ameliorating apical margin positivity during robotic radical prostatectomy. BJU Int. 2010;106(9):1364-1373.

31. Preston MA, Carrière M, Raju G, et al. The prognostic significance of capsular incision into tumor during radical prostatectomy. Eur Urol. 2011;59(4):613-618.

32. Wieder JA, Soloway MS. Incidence, etiology, location, prevention and treatment of positive surgical margins after radical prostatectomy for prostate cancer. J Urol. 1998;160(2):299-315.

33. Secin FP, Serio A, Bianco FJ, et al. Preoperative and intraoperative risk factors for side-specific positive surgical margins in laparoscopic radical prostatectomy for prostate cancer. Eur Urol. 2007;51(3):764-771.

34. Yossepowitch O, Briganti A, Eastham JA, et al. Positive surgical margins after radical prostatectomy: a systematic review and contemporary update. Eur Urol. 2014;65(2):303-313.

35. Eichelberg C, Erbersdobler A, Haese A, et al. Frozen section for the management of intraoperatively detected palpable tumor lesions during nerve-sparing scheduled radical prostatectomy. Eur Urol. 2006;49(6):1011-1018.

36. Kakiuchi Y, Choy B, Gordetsky J, et al. Role of frozen section analysis of surgical margins during robot-assisted laparoscopic radical prostatectomy: a 2608-case experience. Hum Pathol. 2013;44(8):1556-1562.

37. Schlomm T, Tennstedt P, Huxhold C, et al. Neurovascular structureadjacent frozen-section examination (NeuroSAFE) increases nervesparing frequency and reduces positive surgical margins in open and robot-assisted laparoscopic radical prostatectomy: experience after 11,069 consecutive patients. Eur Urol. 2012;62(2):333-340.

38. Hedvig H, Liang W, Wei DC, et al. The role of preoperative endorectal magnetic resonance imaging in the decision regarding whether to preserve or resect neurovascular bundles during radical retropubic prostatectomy. Cancer. 2004;100(12):2655-2663.

39. Ukimura O, Magi Galluzzi C, Gill IS. Real-Time Transrectal Ultrasound Guidance During Laparoscopic Radical Prostatectomy: Impact on Surgical Margins. J Urol. 2006;175(4):1304-1310.

40. Long JA, Lee BH, Guillotreau J, et al. Real-Time Robotic Transrectal Ultrasound Navigation During Robotic Radical Prostatectomy: Initial Clinical Experience. Urology. 2012;80(3):608-613.

41. Wiegel T, Bottke D, Steiner U, et al. Phase III postoperative adjuvant radiotherapy after radical prostatectomy compared with radical prostatectomy alone in pT3 prostate cancer with postoperative undetectable prostate-specific antigen: ARO 96-02/AUO AP 09/95. J Clin Oncol. 2009;27(18):2924-2930.

42. Thompson IM, Tangen CM, Paradelo J, et al. Adjuvant radiotherapy for pathological T3N0M0 prostate cancer significantly reduces risk of metastases and improves survival: long-term follow up of a randomized clinical trial. J Urol. 2009;181(3):956-962. 
43. Thompson IM, Valicenti RK, Albertsen P, et al. Adjuvant and salvage radiotherapy after prostatectomy: AUA/ASTRO Guideline. J Urol. 2013;190(2):441-449.
44. Messing EM, Manola J, Yao J, et al. Immediate versus deferred androgen deprivation treatment in patients with node-positive prostate cancer after radical prostatectomy and pelvic lymphadenectomy. Lancet Oncol. 2006;7(6):472-479. 